\title{
FURTHER GENERALIZATION OF GENERALIZED QUASILINEARIZATION METHOD
}

\author{
V. LAKSHMIKANTHAM and N. SHAHZAD ${ }^{1}$ \\ Florida Institute of Technology \\ Program of Applied Mathematics \\ Melbourne, FL 32901 USA
}

(Received June, 1994; revised August, 1994)

\begin{abstract}
The question whether it is possible to develop monotone sequences that converge to the solution quadratically when the function involved in the initial value problem admits a decomposition into a sum of two functions, is answered positively. This extends the method of generalized quasilinearization to a large class.
\end{abstract}

Key words: Quasilinearization Method, Monotone Sequence, Quadratic Convergence.

AMS (MOS) subject classifications: $34 \mathrm{~A} 34,34 \mathrm{~A} 40$.

\section{Introduction}

It is well known $[1,2]$ that the method of quasilinearization offers an approach for obtaining approximate solutions to nonlinear differential equations.

Consider the IVP

$$
x^{\prime}=f(t, x), x(0)=x_{0} \text { on } J=[0, T] .
$$

If $f(t, x)$ is uniformly convex in $x$ for all $t \in[0, T]$, then the method of quasilinearization provides a monotone increasing sequence of approximate solutions that converges quadratically to the unique solution. Moreover, the sequence provides lower bounds for the solution. Recently, the method of quasilinearization has received much attention after the publication of [9]. Since then, there has been a lot of activity in this area and several interesting results have appeared (see, for example, $[4,5,6,7,8,10,11])$.

In this paper, we show that it is possible to develop monotone sequences that converge to the solution quadratically when $f$ admits a decomposition into a sum of two functions $F$ and $G$ with $F+\psi$ concave and $G+\phi$ convex for some concave function $\psi$ and for some convex function $\phi$. Theorem 2.1 extends Theorem 3.1 in [7] in the setup of [6]. However, we follow the direct approach discovered in [5] rather than the complicated multistage algorithmic method reported in [6]. We do not consider the corresponding results given in [7] that are generated by assuming various coupled upper and lower solutions with suitable extra assumption to avoid monotony.

\footnotetext{
${ }^{1}$ Permanent address: Department of Mathematics, Quaid-i-Azam University, Islamabad, Pakistan.

Printed in the U.S.A. (C)1994 by North Atlantic Science Publishing Company 
Clearly, based on our results and those of [7], it is not difficult to construct the proofs of other possible combinations.

\section{Main Result}

Consider the initial value problem (IVP)

$$
x^{\prime}=f(t, x), \quad x(0)=x_{0}, \quad t \in J=[0, T]
$$

where $f \in C[J \times \mathbb{R}, \mathbb{R}]$. Let $\alpha_{0}, \beta_{0} \in C^{\prime}[J, \mathbb{R}]$ such that $\alpha_{0} \leq \beta_{0}$ on $J$. We define a set

$$
\Omega=\left\{(t, x): \alpha_{0}(t) \leq x \leq \beta_{0}(t), t \in J\right\} .
$$

Theorem 2.1: Assume that

$\left.A_{1}\right) \quad \alpha_{0}, \beta_{0} \in C^{\prime}[J, \mathbb{R}]$ such that $\alpha_{0}^{\prime} \leq f\left(t, \alpha_{0}\right), \beta_{0}^{\prime} \geq f\left(t, \beta_{0}\right)$ and $\alpha_{0} \leq \beta_{0}$ on $J$;

$\left.A_{2}\right) \quad f \in C[\Omega, \mathbb{R}], f$ admits a decomposition $f=F+G$ where $F_{x}, G_{x}, F_{x x}, G_{x x}$ exist and are continuous satisfying $F_{x x}(t, x)+\psi_{x x}(t, x) \leq 0$ and $G_{x x}(t, x)+\phi_{x x}(t, x) \geq 0$ on $\Omega$, where $\phi, \psi \in C[\Omega, \mathbb{R}], \phi_{x}(t, x), \psi_{x}(t, x), \phi_{x x}(t, x), \psi_{x x}(t, x)$ exist, are continuous and $\psi_{x x}(t, x)<0, \phi_{x x}(t, x)>0$ on $\Omega$.

Then there exist monotone sequences $\left\{\alpha_{n}(t)\right\},\left\{\beta_{n}(t)\right\}$ which converge uniformly to the unique solution of (2.1) and the convergence is quadratic.

Proof: In view of $\left(A_{2}\right)$, we see that

$$
F(t, y) \leq H(t, x)-H_{x}(t, x)(x-y)-\psi(t, y)
$$

and

$$
G(t, x) \geq M(t, y)+M_{x}(t, y)(x-y)-\phi(t, x)
$$

for $x \geq y, x, y \in \Omega$, where $H(t, x)=F(t, x)+\psi(t, x)$ and $M(t, x)=G(t, x)+\phi(t, x)$. Also, it is clear that whenever $\alpha_{0}(t) \leq x_{2} \leq x_{1} \leq \beta_{0}(t)$

$$
f\left(t, x_{1}\right)-f\left(t, x_{2}\right) \leq L\left(x_{1}-x_{2}\right), \text { for some } L>0 .
$$

Let $\alpha_{1}, \beta_{1}$ be the solutions of linear IVPs

$$
\alpha_{1}^{\prime}=f\left(t, \alpha_{0}\right)+\left[M_{x}\left(t, \alpha_{0}\right)+H_{x}\left(t, \beta_{0}\right)-\psi_{x}\left(t, \alpha_{0}\right)-\phi_{x}\left(t, \beta_{0}\right)\right]\left(\alpha_{1}-\alpha_{0}\right), \alpha_{1}(0)=x_{0},
$$

and

$$
\beta_{1}^{\prime}=f\left(t, \beta_{0}\right)+\left[M_{x}\left(t, \alpha_{0}\right)+H_{x}\left(t, \beta_{0}\right)-\psi_{x}\left(t, \alpha_{0}\right)-\phi_{x}\left(t, \beta_{0}\right)\right]\left(\beta_{1}-\beta_{0}\right), \beta_{1}(0)=x_{0}
$$

where $\alpha_{0}(0) \leq x_{0} \leq \beta_{0}(0)$.

We shall prove that $\alpha_{0} \leq \alpha_{1}$ on $J$. To do this, let $p=\alpha_{0}-\alpha_{1}$ so that $p(0) \leq 0$. Then

$$
\begin{aligned}
p^{\prime} & =\alpha_{0}^{\prime}-\alpha_{1}^{\prime} \\
& \leq f\left(t, \alpha_{0}\right)-\left[f\left(t, \alpha_{0}\right)+\left\{M_{x}\left(t, \alpha_{0}\right)+H_{x}\left(t, \beta_{0}\right)-\psi_{x}\left(t, \alpha_{0}\right)-\phi_{x}\left(t, \beta_{0}\right)\right\}\left(\alpha_{1}-\alpha_{0}\right)\right] \\
& =\left[M_{x}\left(t, \alpha_{0}\right)+H_{x}\left(t, \beta_{0}\right)-\psi_{x}\left(t, \alpha_{0}\right)-\phi_{x}\left(t, \beta_{0}\right)\right] p .
\end{aligned}
$$


Since $p(0) \leq 0$, we get $\alpha_{0}(t) \leq \alpha_{1}(t)$ on $J$. Next we let $p=\alpha_{1}-\beta_{0}$ and note that $p(0) \leq 0$. Now

$$
\begin{aligned}
p^{\prime} & =\alpha_{1}^{\prime}-\beta_{0}^{\prime} \\
& \leq f\left(t, \alpha_{0}\right)+\left[M_{x}\left(t, \alpha_{0}\right)+H_{x}\left(t, \beta_{0}\right)-\psi_{x}\left(t, \alpha_{0}\right)-\phi_{x}\left(t, \beta_{0}\right)\right]\left(\alpha_{1}-\alpha_{0}\right)-f\left(t, \beta_{0}\right)
\end{aligned}
$$

But since $\beta_{0} \geq \alpha_{0}$, using $(2.2)$ and $\left(A_{2}\right)$, we have

and

$$
F\left(t, \alpha_{0}\right) \leq H\left(t, \beta_{0}\right)-H_{x}\left(t, \beta_{0}\right)\left(\beta_{0}-\alpha_{0}\right)-\psi\left(t, \alpha_{0}\right)
$$

$$
G\left(t, \beta_{0}\right) \geq M\left(t, \alpha_{0}\right)+M_{x}\left(t, \alpha_{0}\right)\left(\beta_{0}-\alpha_{0}\right)-\phi\left(t, \beta_{0}\right)
$$

or

$$
F\left(t, \alpha_{0}\right) \leq F\left(t, \beta_{0}\right)-H_{x}\left(t, \beta_{0}\right)\left(\beta_{0}-\alpha_{0}\right)+\left[\psi\left(t, \beta_{0}\right)-\psi\left(t, \alpha_{0}\right)\right]
$$

and

$$
G\left(t, \beta_{0}\right) \geq G\left(t, \alpha_{0}\right)+M_{x}\left(t, \alpha_{0}\right)\left(\beta_{0}-\alpha_{0}\right)-\left[\phi\left(t, \beta_{0}\right)-\phi\left(t, \alpha_{0}\right)\right] .
$$

Now, by using the mean value theorem,

$$
\psi\left(t, \beta_{0}\right)-\psi\left(t, \alpha_{0}\right)=\psi_{x}(t, \xi)\left(\beta_{0}-\alpha_{0}\right)
$$

and

$$
\phi\left(t, \beta_{0}\right)-\phi\left(t, \alpha_{0}\right)=\phi_{x}(t, \delta)\left(\beta_{0}-\alpha_{0}\right)
$$

where $\alpha_{0}<\xi, \delta<\beta_{0}$. Because $\psi_{x}(t, x)$ is decreasing in $x$ and $\phi_{x}(t, x)$ is increasing in $x$, it follows that

and

$$
\psi\left(t, \beta_{0}\right)-\psi\left(t, \alpha_{0}\right) \leq \psi_{x}\left(t, \alpha_{0}\right)\left(\beta_{0}-\alpha_{0}\right)
$$

$$
\phi\left(t, \beta_{0}\right)-\phi\left(t, \alpha_{0}\right) \leq \phi_{x}\left(t, \beta_{0}\right)\left(\beta_{0}-\alpha_{0}\right)
$$

Thus we get

$$
F\left(t, \alpha_{0}\right) \leq F\left(t, \beta_{0}\right)-\left[H_{x}\left(t, \beta_{0}\right)-\psi_{x}\left(t, \alpha_{0}\right)\right]\left(\beta_{0}-\alpha_{0}\right)
$$

and

$$
G\left(t, \beta_{0}\right) \geq G\left(t, \alpha_{0}\right)+\left[M_{x}\left(t, \alpha_{0}\right)-\phi_{x}\left(t, \beta_{0}\right)\right]\left(\beta_{0}-\alpha_{0}\right)
$$

which in turn yields

$$
p^{\prime} \leq\left[M_{x}\left(t, \alpha_{0}\right)+H_{x}\left(t, \beta_{0}\right)-\psi_{x}\left(t, \alpha_{0}\right)-\phi_{x}\left(t, \beta_{0}\right)\right] p .
$$

Consequently, $p(t) \leq 0$ on $J$ proving $\alpha_{1} \leq \beta_{0}$ on $J$. 
As a result, we have

$$
\alpha_{0}(t) \leq \alpha_{1}(t) \leq \beta_{0}(t) \text { on } J
$$

Similarly, we can show that $\alpha_{0}(t) \leq \beta_{1}(t) \leq \beta_{0}(t)$ on $J$. We need to prove that $\alpha_{1}(t) \leq \beta_{1}(t)$ on $J$ so that it follows

$$
\alpha_{0}(t) \leq \alpha_{1}(t) \leq \beta_{1}(t) \leq \beta_{0}(t) \text { on } J
$$

Since $\alpha_{0} \leq \alpha_{1} \leq \beta_{0}$, using (2.2) and (2.4), we see that

$$
\begin{aligned}
& \alpha_{1}^{\prime}=f\left(t, \alpha_{0}\right)+\left[M_{x}\left(t, \alpha_{0}\right)+H_{x}\left(t, \beta_{0}\right)-\psi_{x}\left(t, \alpha_{0}\right)-\phi_{x}\left(t, \beta_{0}\right)\right]\left(\alpha_{1}-\alpha_{0}\right) \\
& =\left[F\left(t, \alpha_{0}\right)+H_{x}\left(t, \beta_{0}\right)\left(\alpha_{1}-\alpha_{0}\right)\right]+\left[G\left(t, \alpha_{0}\right)+M_{x}\left(t, \alpha_{0}\right)\left(\alpha_{1}-\alpha_{0}\right)\right] \\
& -\psi_{x}\left(t, \alpha_{0}\right)\left(\alpha_{1}-\alpha_{0}\right)-\phi_{x}\left(t, \beta_{0}\right)\left(\alpha_{1}-\alpha_{0}\right) \\
& \leq\left[F\left(t, \alpha_{0}\right)+H_{x}\left(t, \alpha_{1}\right)\left(\alpha_{1}-\alpha_{0}\right)\right]+\left[G\left(t, \alpha_{0}\right)+M_{x}\left(t, \alpha_{0}\right)\left(\alpha_{1}-\alpha_{0}\right)\right] \\
& -\psi_{x}\left(t, \alpha_{0}\right)\left(\alpha_{1}-\alpha_{0}\right)-\phi_{x}\left(t, \beta_{0}\right)\left(\alpha_{1}-\alpha_{0}\right) \\
& \leq\left[F\left(t, \alpha_{1}\right)+\psi\left(t, \alpha_{1}\right)-\psi\left(t, \alpha_{0}\right)\right]+\left[G\left(t, \alpha_{1}\right)+\phi\left(t, \alpha_{1}\right)-\phi\left(t, \alpha_{0}\right)\right] \\
& -\psi_{x}\left(t, \alpha_{0}\right)\left(\alpha_{1}-\alpha_{0}\right)-\phi_{x}\left(t, \beta_{0}\right)\left(\alpha_{1}-\alpha_{0}\right) \\
& \leq f\left(t, \alpha_{1}\right)+\psi_{x}\left(t, \alpha_{0}\right)\left(\alpha_{1}-\alpha_{0}\right)+\phi_{x}\left(t, \beta_{0}\right)\left(\alpha_{1}-\alpha_{0}\right) \\
& -\psi_{x}\left(t, \alpha_{0}\right)\left(\alpha_{1}-\alpha_{0}\right)-\phi_{x}\left(t, \beta_{0}\right)\left(\alpha_{1}-\alpha_{0}\right) \\
& =f\left(t, \alpha_{1}\right) \text {. }
\end{aligned}
$$

Here we have used the mean value theorem and the facts that $H_{x}(t, x), \psi_{x}(t, x)$ are decreasing in $x$ and $\phi_{x}(t, x)$ is increasing in $x$.

Similarly, we can prove that $\beta_{1}^{\prime} \geq f\left(t, \beta_{1}\right)$ and therefore by Theorem 1.1.1 [3], it follows that $\alpha_{1}(t) \leq \beta_{1}(t)$ on $J$ which shows that $(2.6)$ is valid.

Assume now that for some $k>1, \alpha_{k}^{\prime} \leq f\left(t, \alpha_{k}\right), \beta_{k}^{\prime} \geq f\left(t, \beta_{k}\right)$ and $\alpha_{k}(t) \leq \beta_{k}(t)$ on $J$, we shall show that

$$
\alpha_{k}(t) \leq \alpha_{k+1}(t) \leq \beta_{k+1}(t) \leq \beta_{k}(t) \text { on } J
$$

where $\alpha_{k+1}$ and $\beta_{k+1}$ are the solutions of linear IVP's

$$
\begin{gathered}
\alpha_{k+1}^{\prime}=f\left(t, \alpha_{k}\right)+\left[M_{x}\left(t, \alpha_{k}\right)+H_{x}\left(t, \beta_{k}\right)-\psi_{x}\left(t, \alpha_{k}\right)-\phi_{x}\left(t, \beta_{k}\right)\right]\left(\alpha_{k+1}-\alpha_{k}\right), \\
\alpha_{k+1}(0)=x_{0},
\end{gathered}
$$

and

$$
\beta_{k+1}^{\prime}=f\left(t, \beta_{k}\right)+\left[M_{x}\left(t, \alpha_{k}\right)+H_{x}\left(t, \beta_{k}\right)-\psi_{x}\left(t, \alpha_{k}\right)-\phi_{x}\left(t, \beta_{k}\right)\right]\left(\beta_{k+1}-\beta_{k}\right),
$$




$$
\beta_{k+1}(0)=x_{0}
$$

Hence setting $p=\alpha_{k}-\alpha_{k+1}$, it follows as before that

$$
p^{\prime} \leq\left[M_{x}\left(t, \alpha_{k}\right)+H_{x}\left(t, \beta_{k}\right)-\psi_{x}\left(t, \alpha_{k}\right)-\phi_{x}\left(t, \beta_{k}\right)\right] p \text { on } J
$$

and $p(0)=0$ which again implies $p(t) \leq 0$ on $J$. On the other hand, letting $p=\alpha_{k+1}-\beta_{k}$ yields as before $p^{\prime} \leq\left[M_{x}\left(t, \alpha_{k}\right)+H_{x}\left(t, \beta_{k}\right)-\psi_{x}\left(t, \alpha_{k}\right)-\phi_{x}\left(t, \beta_{k}\right)\right] p$. This proves that $p(t) \leq 0$, since $p(0)=0$ and therefore we have $\alpha_{k} \leq \alpha_{k+1} \leq \beta_{k}$ on $J$. In a similar manner, we can prove that

$$
\alpha_{k} \leq \beta_{k+1} \leq \beta_{k} \text { on } J .
$$

Now using (2.2), (2.8) and the fact $\beta_{k} \geq \alpha_{k+1} \geq \alpha_{k}$, we get

$$
\begin{aligned}
\alpha_{k+1}^{\prime} & =f\left(t, \alpha_{k}\right)+\left[M_{x}\left(t, \alpha_{k}\right)+H_{x}\left(t, \beta_{k}\right)-\psi_{x}\left(t, \alpha_{k}\right)-\phi_{x}\left(t, \beta_{k}\right)\right]\left(\alpha_{k+1}-\alpha_{k}\right) \\
& =\left[F\left(t, \alpha_{k}\right)+H_{x}\left(t, \beta_{k}\right)\left(\alpha_{k+1}-\alpha_{k}\right)\right]+\left[G\left(t, \alpha_{k}\right)+M_{x}\left(t, \alpha_{k}\right)\left(\alpha_{k+1}-\alpha_{k}\right)\right] \\
& \left.-\psi_{x}\left(t, \alpha_{k}\right)\left(\alpha_{k+1}-\alpha_{k}\right)-\phi_{x}\left(t, \beta_{k}\right)\left(\alpha_{k+1}-\alpha_{k}\right)\right] \\
& \leq\left[F\left(t, \alpha_{k}\right)+H_{x}\left(t, \alpha_{k+1}\right)\left(\alpha_{k+1}-\alpha_{k}\right)\right]+\left[G\left(t, \alpha_{k}\right)+M_{x}\left(t, \alpha_{k}\right)\left(\alpha_{k+1}-\alpha_{k}\right)\right] \\
& -\psi\left(t, \alpha_{k}\right)\left(\alpha_{k+1}-\alpha_{k}\right)-\phi_{x}\left(t, \beta_{k}\right)\left(\alpha_{k+1}-\alpha_{k}\right) \\
& \leq\left[F\left(t, \alpha_{k+1}\right)+\psi\left(t, \alpha_{k+1}\right)-\psi\left(t, \alpha_{k}\right)\right]+\left[G\left(t, \alpha_{k+1}\right)+\phi\left(t, \alpha_{k+1}\right)-\phi\left(t, \alpha_{k}\right)\right] \\
& -\psi_{x}\left(t, \alpha_{k}\right)\left(\alpha_{k+1}-\alpha_{k}\right)-\phi_{x}\left(t, \beta_{k}\right)\left(\alpha_{k+1}-\alpha_{k}\right) \\
& =f\left(t, \alpha_{k+1}\right) .
\end{aligned}
$$

Here we have used the mean value theorem and the facts that $H_{x}(t, x), \psi_{x}(t, x)$ are decreasing in $x$ and $\phi_{x}(t, x)$ is increasing in $x$.

Similar arguments yield $\beta_{k+1}^{\prime} \geq f\left(t, \beta_{k+1}\right)$ and hence Theorem 1.1.1 [3] shows that $\alpha_{k+1}(t) \leq \beta_{k+1}(t)$ on $J$ which proves (2.7).

Hence we obtain by induction

$$
\alpha_{0} \leq \alpha_{1} \leq \alpha_{2} \leq \ldots \leq \alpha_{n} \leq \beta_{n} \leq \ldots \leq \beta_{2} \leq \beta_{1} \leq \beta_{0} \text { on } J
$$

Now using standard arguments, it is easy to show that the sequences $\left\{\alpha_{n}(t)\right\},\left\{\beta_{n}(t)\right\}$ converge uniformly and monotonically to the unique solution $x(t)$ of $(2.1)$ on $J$. Finally, to prove quadratic convergence, we consider

$$
p_{n+1}(t)=x(t)-\alpha_{n+1}(t) \geq 0, \quad q_{n+1}(t)=\beta_{n+1}(t)-x(t) \geq 0 .
$$

Note that $p_{n+1}(0)=0=q_{n+1}(0)$. We then have

$$
\begin{aligned}
& p_{n+1}^{\prime}=x^{\prime}-\alpha_{n+1}^{\prime} \\
& \quad=f(t, x)-\left[f\left(t, \alpha_{n}\right)+\left\{M_{x}\left(t, \alpha_{n}\right)+H_{x}\left(t, \beta_{n}\right)-\psi_{x}\left(t, \alpha_{n}\right)-\phi_{x}\left(t, \beta_{n}\right)\right]\left(\alpha_{n+1}-\alpha_{n}\right)\right]
\end{aligned}
$$




$$
\begin{aligned}
& =\left\{H(t, x)-H\left(t, \alpha_{n}\right)\right\}+\left\{M(t, x)-M\left(t, \alpha_{n}\right)\right\}-\left\{\psi(t, x)-\psi\left(t, \alpha_{n}\right)\right\} \\
& \left.-\left\{\phi(t, x)-\phi\left(t, \alpha_{n}\right)\right\}-\left\{M_{x}\left(t, \alpha_{n}\right)+H_{x}\left(t, \beta_{n}\right)-\psi_{x}\left(t, \alpha_{n}\right)-\phi_{x}\left(t, \beta_{n}\right)\right\}\left(\alpha_{n+1}-\alpha_{n}\right)\right] \\
& \leq H_{x}\left(t, \alpha_{n}\right)\left(x-\alpha_{n}\right)+M_{x}(t, x)\left(x-\alpha_{n}\right)-\psi_{x}(t, x)\left(x-a_{n}\right)-\phi_{x}\left(t, \alpha_{n}\right)\left(x-\alpha_{n}\right) \\
& -\left\{M_{x}\left(t, \alpha_{n}\right)+H_{x}\left(t, \beta_{n}\right)-\psi_{x}\left(t, \alpha_{n}\right)-\phi_{x}\left(t, \beta_{n}\right)\right\}\left(\alpha_{n+1}-x+x-\alpha_{n}\right) \\
& =H_{x x}(t, \xi)\left(\alpha_{n}-\beta_{n}\right)\left(x-\alpha_{n}\right)+M_{x x}(t, \delta)\left(x-\alpha_{n}\right)^{2}-\psi_{x x}(t, \gamma)\left(x-\alpha_{n}\right)^{2} \\
& +\phi_{x x}(t, \theta)\left(\beta_{n}-\alpha_{n}\right)\left(x-\alpha_{n}\right)+\left\{M_{x}\left(t, \alpha_{n}\right)+H_{x}\left(t, \beta_{n}\right)-\psi_{x}\left(t, \alpha_{n}\right)-\phi_{x}\left(t, \beta_{n}\right)\right\} p_{n+1} \\
& =\left\{\phi_{x x}(t, \theta)-H_{x x}(t, \xi)\right\} p_{n}\left(q_{n}+p_{n}\right)+\left\{M_{x x}(t, \delta)-\psi_{x x}(t, \gamma)\right\} p_{n}^{2} \\
& +\left\{M_{x}\left(t, \alpha_{n}\right)+H_{x}\left(t, \beta_{n}\right)-\psi_{x}\left(t, \alpha_{n}\right)-\phi_{x}\left(t, \beta_{n}\right)\right\} p_{n+1} \\
& \leq\left\{\phi_{x x}(t, \theta)-H_{x x}(t, \xi)\right\} p_{n}\left(q_{n}+p_{n}\right)+\left\{M_{x x}(t, \delta)-\psi_{x x}(t, \gamma)\right\} p_{n}^{2} \\
& +\left\{G_{x}\left(t, \alpha_{n}\right)+F_{x}\left(t, \beta_{n}\right)\right\} p_{n+1}
\end{aligned}
$$

where $\alpha_{n}<\xi, \theta<\beta_{n}$ and $\alpha_{n}<\delta, \gamma<x$.

Hence, we obtain

$$
\begin{gathered}
p_{n+1}^{\prime} \leq(A+B+C) p_{n}\left(q_{n}+p_{n}\right)+(A+C+D) p_{n}^{2}+(E+K) p_{n+1} \\
\leq(A+B+C)\left(2 p_{n}^{2}+q_{n}^{2}\right)+(A+C+D) p_{n}^{2}+(E+K) p_{n+1} \\
=Q p_{n}^{2}+R q_{n}^{2}+S p_{n+1}
\end{gathered}
$$

where on $\Omega$,

$$
\begin{gathered}
\left|\phi_{x x}(t, x)\right|<A,\left|F_{x x}(t, x)\right|<B,\left|\psi_{x x}(t, x)\right|<C,\left|G_{x x}(t, x)\right|<D, \\
\left|G_{x}(t, x)\right|<E,\left|F_{x}(t, x)\right|<K, Q=3 A+2 B+3 C+D, R=A+B+C
\end{gathered}
$$

and

$$
S=E+K \text {. }
$$

Now Gronwall's inequality implies

$$
0 \leq p_{n+1}(t) \leq \int_{0}^{t} e^{S(t-s)}\left[Q p_{n}^{2}(s)+R q_{n}^{2}(s)\right] d s \text { on } J
$$

which yield the desired result

$$
\max _{J}\left|x(t)-\alpha_{n+1}(t)\right| \leq \frac{e^{S T}}{S}\left[Q \max _{J}\left|x(t)-\alpha_{n}(t)\right|^{2}+R \max _{J}\left|\beta_{n}(t)-x(t)\right|^{2}\right] .
$$

Similarly,

$$
\begin{aligned}
& q_{n+1}^{\prime}=\beta_{n+1}^{\prime}-x^{\prime} \\
& \quad=f\left(t, \beta_{n}\right)+\left[M_{x}\left(t, \alpha_{n}\right)+H_{x}\left(t, \beta_{n}\right)-\psi_{x}\left(t, \alpha_{n}\right)-\phi_{x}\left(t, \beta_{n}\right)\right]\left(\beta_{n+1}-\beta_{n}\right)-f(t, x)
\end{aligned}
$$




$$
\begin{aligned}
& =\left\{H\left(t, \beta_{n}\right)-H(t, x)\right\}+\left\{M\left(t, \beta_{n}\right)-M(t, x)\right\}+\left\{\psi(t, x)-\psi\left(t, \beta_{n}\right)\right\} \\
& +\left\{\phi(t, x)-\phi\left(t, \beta_{n}\right)\right\}+\left\{M_{x}\left(t, \alpha_{n}\right)+H_{x}\left(t, \beta_{n}\right)-\psi_{x}\left(t, \alpha_{n}\right)-\phi_{x}\left(t, \beta_{n}\right)\right\}\left(\beta_{n+1}-\beta_{n}\right) \\
& \leq H_{x}(t, x)\left(\beta_{n}-x\right)+M_{x}\left(t, \beta_{n}\right)\left(\beta_{n}-x\right)-\psi_{x}\left(t, \beta_{n}\right)\left(\beta_{n}-x\right)-\phi_{x}(t, x)\left(\beta_{n}-x\right) \\
& +\left\{M_{x}\left(t, \alpha_{n}\right)+H_{x}\left(t, \beta_{n}\right)-\psi_{x}\left(t, \alpha_{n}\right)-\phi_{x}\left(t, \beta_{n}\right)\right\}\left(\beta_{n+1}-x-x-\beta_{n}\right) \\
& \leq\left\{\phi_{x x}(t, \theta)-H_{x x}(t, \xi)\right\}\left(\beta_{n}-x\right)^{2}+\left\{M_{x x}(t, \delta)-\psi_{x x}(t, \gamma)\right\}\left(\beta_{n}-\alpha_{n}\right)\left(\beta_{n}-x\right) \\
& +\left\{G_{x}\left(t, \alpha_{n}\right)+F_{x}\left(t, \beta_{n}\right)\right\}\left(\beta_{n+1}-x\right) \\
& =\left\{\phi_{x x}(t, \theta)-H_{x x}(t, \xi)\right\} q_{n}^{2}+\left\{M_{x x}(t, \delta)-\psi_{x x}(t, \gamma)\right\}\left(q_{n}+p_{n}\right) q_{n} \\
& +\left\{G_{x}\left(t, \alpha_{n}\right)+F_{x}\left(t, \beta_{n}\right)\right\} q_{n+1}
\end{aligned}
$$

where $\alpha_{n}<\xi, \theta<\beta_{n}$ and $\alpha_{n}<\delta, \gamma<x$.

Hence, we obtain

$$
\begin{aligned}
& q_{n+1}^{\prime} \leq(A+B+C) q_{n}^{2}(A+C+D) q_{n}\left(q_{n}+p_{n}\right)+(E+K) q_{n+1} \\
& \quad \leq(A+B+C) q_{n}^{2}+(A+C+D)\left(2 q_{n}^{2}+p_{n}^{2}\right)+(E+K) q_{n+1} \\
& \quad=Q^{*} q_{n}^{2}+R^{*} p_{n}^{2}+S q_{n+1}
\end{aligned}
$$

where the constants $A, B, C, D, E, K$ and $S$ are as in (2.10) and $Q^{*}=3 A+B+3 C+2 D$, $R^{*}=A+C+D$.

An application of Gronwall's inequality yields

and hence

$$
0 \leq q_{n+1}(t) \leq \int_{0}^{t} e^{S(t-s)}\left[Q^{*} q_{n}^{2}(s)+R^{*} p_{n}^{2}(s)\right] d s \text { on } J
$$

$$
\max _{J}\left|\beta_{n+1}(t)-x(t)\right| \leq \frac{e^{S T}}{S}\left[Q^{*} \max _{J}\left|\beta_{n}(t)-x(t)\right|^{2}+R^{*} \max _{J}\left|x(t)-\alpha_{n}(t)\right|^{2}\right] .
$$

This completes the proof of the theorem.

\section{References}

[1] Bellman, R., Methods of Nonlinear Analysis Vol. II, Academic Press, New York, 1973.

[2] Bellman, R. and Kalaba, R., Quasilinearization and Nonlinear Boundary Value Problems, American Elsevier, New York, 1965.

[3] Ladde, G.S., Lakshmikantham, V., and Vatsala, A.S., Monotone Iterative Techniques for Nonlinear Differential Equations, Pitman, Boston, 1985.

[4] Lakshmikantham, V., An extension of the method of quasilinearization, (to appear in J.O.T.A.).

[5] Lakshmikantham, V., Further improvement to generalized quasilinearization method, (to appear in Nonlinear Analysis). 
[6] Lakshmikantham, V., Leela, S. and McRae, F.A., Improved generalized quasilinearization method, (to appear in Nonlinear Analysis).

[7] Lakshmikantham, V., Leela, S. and Sivasundaram, S., Extensions of the method of quasilinearization, (to appear in J.O.T.A.).

[8] Lakshmikantham, V. and Köksal, S., Another extension of the method of quasilinearization, (to appear in the Proceedings of Dynamic Systems and Applications).

[9] Lakshmikantham, V. and Malek, S., Generalized quasilinearization, Nonlinear World 1 (1994), 59-63.

[10] Malek, S. and Vatsala, A.S., Method of generalized quasilinearization for second order boundary value problem, Inequalities and Applications 3 (1994), (to appear).

[11] McRae, F.A., Generalized quasilinearization of stochastic initial value problems, Stochastic Analysis and Applications 13 (1994), (to appear). 


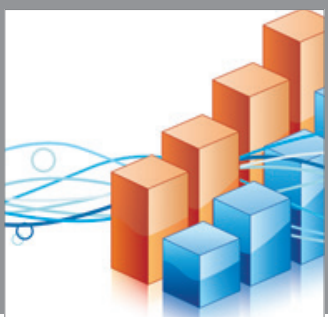

Advances in

Operations Research

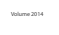

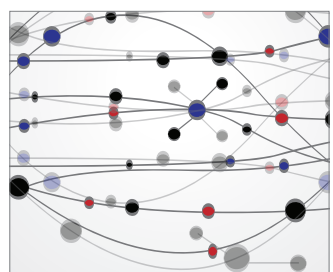

\section{The Scientific} World Journal
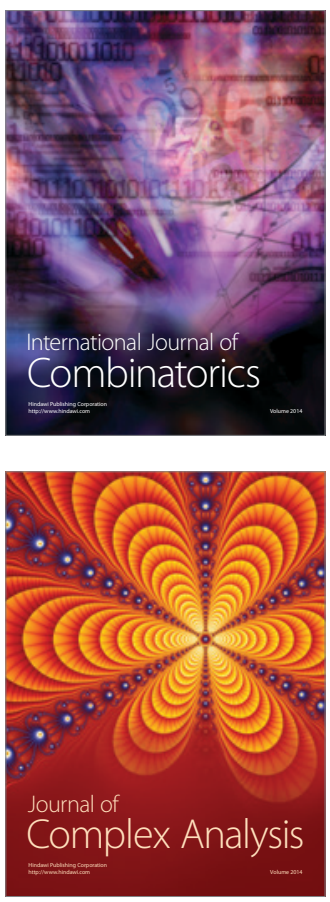

International Journal of

Mathematics and

Mathematical

Sciences
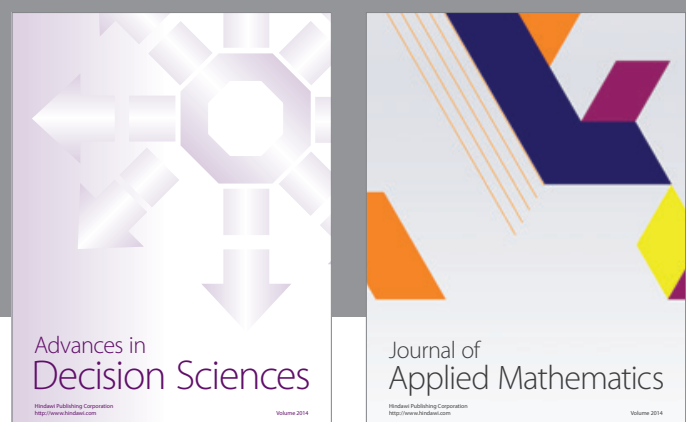

Journal of

Applied Mathematics
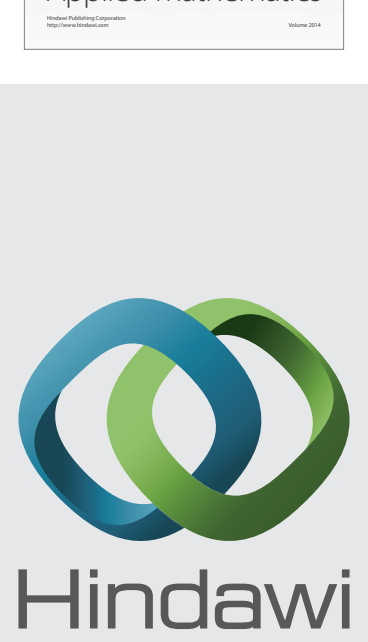

Submit your manuscripts at http://www.hindawi.com
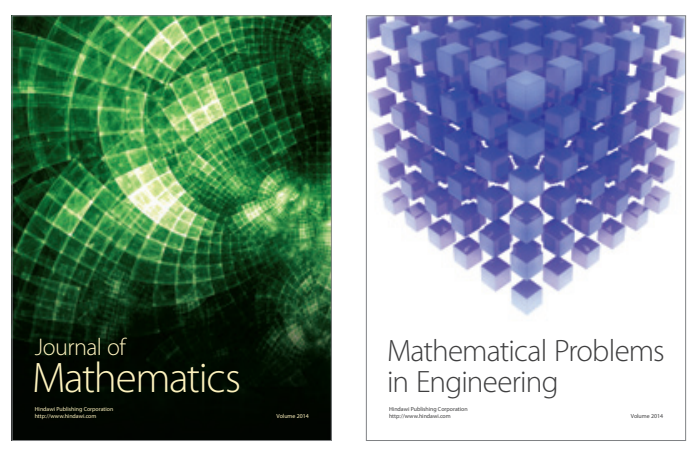

Mathematical Problems in Engineering
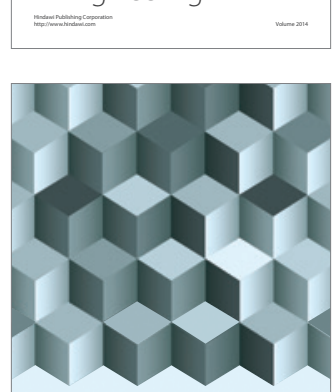

Journal of

Function Spaces
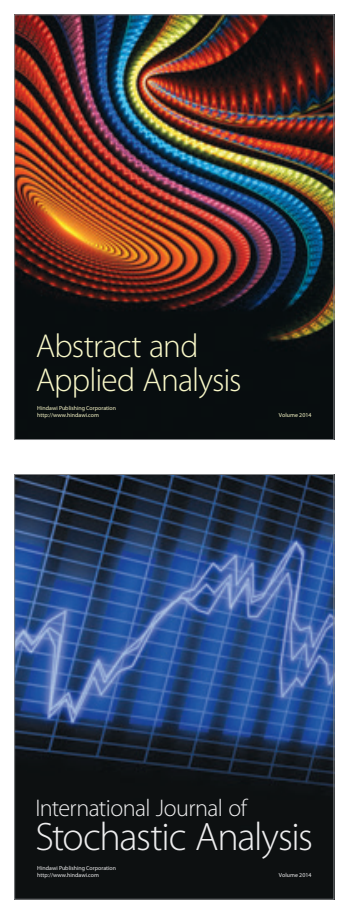

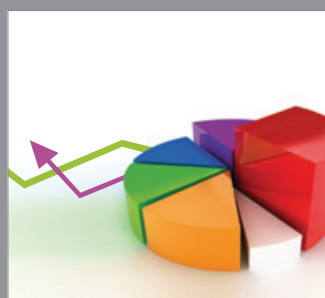

ournal of

Probability and Statistics

Promensencen
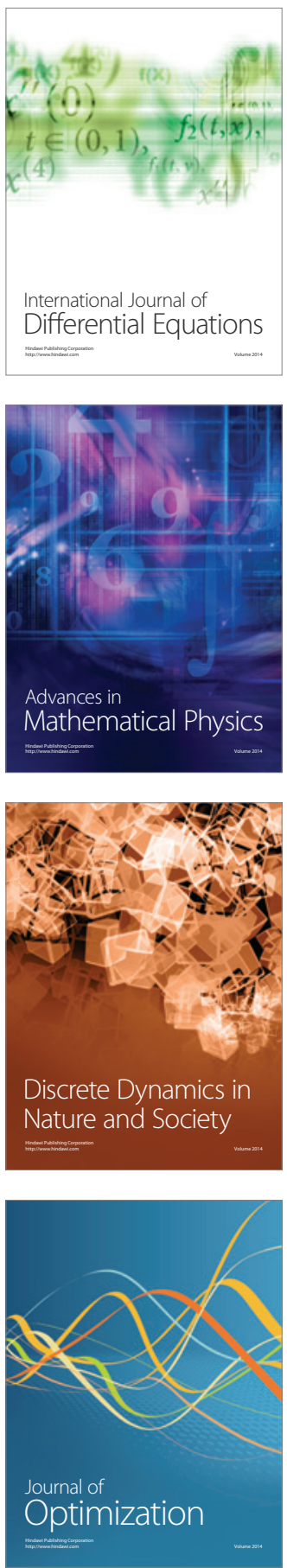